\title{
Correction to: Equilibrium and pricing analysis for an unreliable retrial queue with limited idle period and single vacation
}

\section{Shan Gao ${ }^{1}$ (D) Hua Dong ${ }^{2} \cdot$ Xianchao Wang $^{1}$}

Published online: 18 June 2019

๑) Springer-Verlag GmbH Germany, part of Springer Nature 2019

\section{Correction to: Operational Research https://doi.org/10.1007/s12351-018-0437-7}

In the original publication of the article, the below-mentioned equations had been incorrectly published. The corrected equations are given as follows:

1. Line 240, Eq. (19)

$$
q_{e}= \begin{cases}0, & \frac{R}{C}<L_{e}, \\ \frac{R-C\left(\tau_{1}+\tau_{2}\right)}{\rho\left(R-C \tau_{1}\right)+C \tau_{2} \rho_{3}}, & L_{e} \leq \frac{R}{C} \leq U_{e}, \\ 1, & \frac{R}{C}>U_{e},\end{cases}
$$

2. Line 288 , Eq. (27)

$$
q_{s o c}= \begin{cases}0, & \frac{R}{C}<L_{s o c} \\ \frac{1-x_{2}}{\rho}, & L_{s o c} \leq \frac{R}{C} \leq U_{s o c}, \\ 1, & \frac{R}{C}>U_{s o c}\end{cases}
$$

3. Line 298 , the second inequality

$$
x_{2}=\frac{B+\sqrt{B^{2}+A B\left(\rho+\rho_{3}\right)}}{A}>0
$$

The original article can be found online at https://doi.org/10.1007/s12351-018-0437-7.

Shan Gao

sgao_09@yeah.net

1 School of Mathematics and Statistics, Fuyang Normal College, Fuyang 236037, Anhui, China

2 School of Statistics, Qufu Normal University, Shandong 273165, China 
Publisher's Note Springer Nature remains neutral with regard to jurisdictional claims in published maps and institutional affiliations. 